\title{
Towards milli-Hertz laser frequency noise on a chip
}

\author{
Heming Wang, ${ }^{\dagger}$ Lue Wu, ${ }^{\dagger}$ Zhiquan Yuan, ${ }^{\dagger}$ and Kerry Vahala* ${ }^{*}$ \\ T. J. Watson Laboratory of Applied Physics, California Institute of Technology, Pasadena, California 91125, USA \\ *vahala@caltech.edu
}

\begin{abstract}
A fundamental frequency noise of $9 \mathrm{mHz} \cdot \mathrm{Hz} / \mathrm{Hz}$ is demonstrated in an on-chip Brillouin laser. The noise measurement uses the frequency discrimination method enhanced by cross-correlator. The results set new performance targets for chip-based laser platforms. (c) 2021 The Author(s)
\end{abstract}

Narrow-linewidth lasers are important to many applications spanning precision metrology to sensing systems, and their chip-scale miniaturization is receiving increasing attention. Stimulated Brillouin lasers (SBLs) have emerged as a powerful platform for narrow linewidth on-chip operation [1]. Here, an ultra-low frequency noise level of $9 \mathrm{mHz} \cdot \mathrm{Hz} / \mathrm{Hz}$ in an on-chip SBL is demonstrated. The noise level has a power dependence and magnitude that are consistent with fundamental white frequency noise, and would correspond to a linewidth of $60 \mathrm{mHz}$. This linewidth is $5 \times$ lower than previous SBL reports, and it is measured using an enhancement of the self-heterodyne optical frequency discriminator method [2]. The ultra-low noise level is made possible by the high optical Q of the resonator in combination with higher single-mode laser power through suppression of Brillouin cascade [1,3].

Typically, to satisfy the phase matching condition, SBL microcavities are designed so as to align the freespectral-range (FSR) to the Brillouin shift frequency [5]. However, as the FSR dispersion is often small, a neighboring longitudinal mode can also phase match to the initial Stokes laser wave, leading to lasing cascade [1] (see Fig. 1a, upper panel). Cascading is detrimental to low frequency noise operation as the laser power of the first Stokes wave (now the pumping wave) becomes clamped. Moreover, frequency noise is increased due to the presence of the second-order Stokes wave [4]. Here, by using different transverse mode families for pumping and Stokes waves, cascade is blocked (see Fig. 1a, lower panel) so that Brillouin laser power can be substantially increased, resulting in reduced fundamental frequency noise. The experimental setup is shown in Fig. 1b. An external-cavity diode laser (ECDL) is amplified by an erbium-doped fiber amplifier (EDFA) and sent to a tapered fiber for coupling to the resonator. The Brillouin laser wave, propagating in the opposite direction of the pump wave, is collected with a circulator. An optical spectrum analyzer (OSA) is used to monitor pump and SBL spectra and to ensure that no cascading occurs. To measure the frequency noise, the laser output is sent to an optical frequency discriminator enhanced with a cross correlation method.

A silica wedge resonator is used for the SBL [5]. To identify appropriate mode families for pumping and Stokes lasing waves, mode dispersion measurements are performed (Fig. 2a). The plotted points give $\Delta v_{\mu}^{m} \equiv$ $v_{\mu}^{m}-v_{0}-\mu \times$ FSR, where $v_{\mu}^{m}$ is the frequency of longitudinal mode $\mu$ belonging to transverse mode family $m$. The pump mode must be located one Brillouin shift higher in frequency relative to the Stokes laser mode frequency. This condition, represented by the blue arrow phase-matching line in Fig. 2(a), is wrapped (modulo the
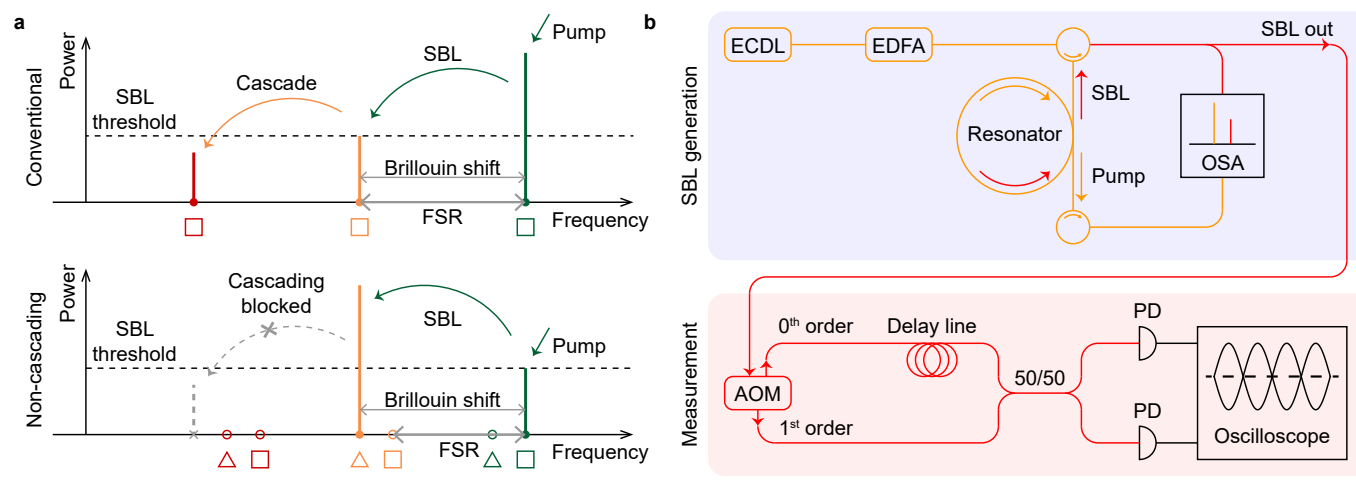

Fig. 1. Operation of Brillouin lasers and experimental setup. a. Upper panel: spectral diagram for conventional SBL operation, where the FSR is matched to the Brillouin frequency shift. Lower panel: spectral diagram for non-cascading laser operation, where FSR is intentionally mismatched from the Brillouin shift. Modes with the same marker shape belong to the same mode family. $\mathbf{b}$. Experimental setup consisting of SBL generation and frequency noise measurement sections. 
(a)

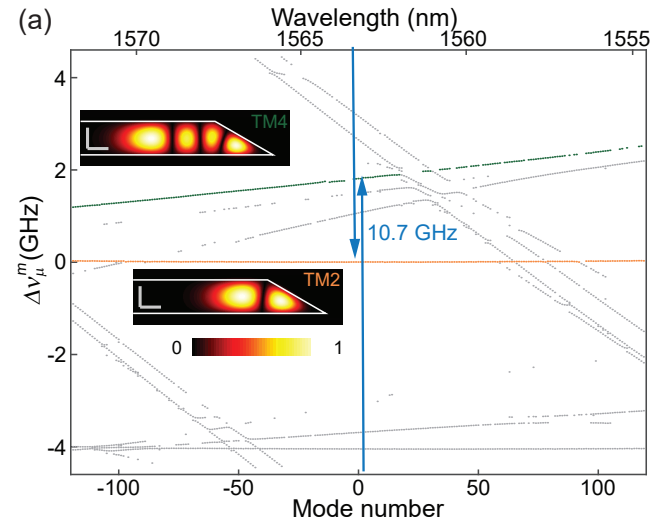

(b)

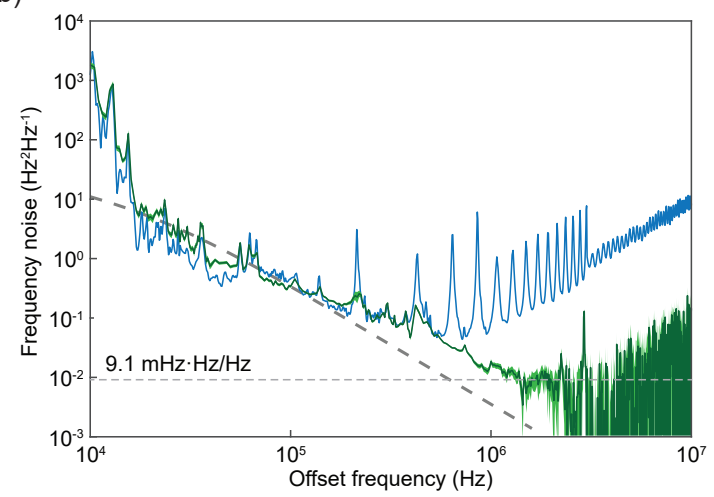

Fig. 2. Brillouin laser frequency noise measurement. a. Measured mode dispersion of the resonator with the SBL (TM2) and pump (TM4) mode families highlighted. The vertical blue arrowed line gives the Brillouin shift. Insets. Simulated modal electric field (norm). Scale bars, $5 \mu \mathrm{m}$. b. SBL frequency noise measured by conventional optical discriminator method in combination with an electrical phase noise analyzer (blue) and by the cross-correlation technique (green). The light green shading shows the error range. The dark gray line shows the simulated thermorefractive noise.

FSR) in making the plot. Two mode families are selected as the pump and SBL modes (green and orange in the figure). Both of these mode families feature high intrinsic Q-factors around 400 million [6] and also experience only a few mode crossings with other mode families.

The self-heterodyne optical frequency discriminator approach is applied to measure the frequency noise spectral density of the generated laser signal (Fig. 2b). However, at high offset frequencies (above $1 \mathrm{MHz}$ ), the frequency noise floor and measurement sensitivity were found to be limited by technical noise from the photodetectors (PD). To overcome this limitation, we applied cross correlation (XCOR) of the electrical signals produced at the interferometer output. The present measurement is distinct in applying XCOR to optical phase noise measurement, and also does not require reference optical or radio frequency oscillators. As shown in Fig. 1b, the measurement setup employs an AOM to split the input light into frequency-shifted ( $1^{\text {st }}$ order output) and un-shifted parts $\left(0^{\text {th }}\right.$ order output), the latter being delayed by a 1-km-long fiber. However, instead of a single photodetector, two photodetectors receive the optical signals. Their outputs are recorded using an oscilloscope for subsequent XCOR to remove detector technical noise [7].

A comparison of the measurements both with and without application of the XCOR method is presented in Fig. 2b. Both traces overlap well at low offset frequencies (below $500 \mathrm{kHz}$ ), where the noise is believed to be thermo-refractive in origin based on simulations shown in the figure. However, at higher offset frequencies, the noise spectra measured using the conventional (non XCOR) approach contain spurs that originate from destructive interference produced by the delay in the interferometer. The noise measurement sensitivity is enhanced by 10 to $15 \mathrm{~dB}$ using the XCOR method to suppress the detector noise. By averaging the white-like portion of the measured frequency noise (between $1.5 \mathrm{MHz}$ to $2 \mathrm{MHz}$ ), this frequency noise level is estimated to be $9 \mathrm{mHz} \cdot \mathrm{Hz} / \mathrm{Hz}$ (equivalent to a Lorentzian linewidth of $60 \mathrm{mHz}$ ). This noise is found to vary inversely with Stokes power and to also agree reasonably well with predictions of the expected fundamental white noise level [1].

These results demonstrate the potential for silica-based high-Q laser platforms to achieve extremely narrow laser linewidths. Even while the noise at low offset frequencies remains high, these noise sources can be suppressed in certain applications such as the Sagnac gyroscope that rely upon relative noise of co-lasing waves [8].

We acknowledge support from AFOSR (FA9550-18-1-0353) and the Caltech Kavli Nanoscience Institute.

\section{References}

1. J. Li, H. Lee, T. Chen \& K. J. Vahala, Opt. Express 20, 20170-20180 (2012).

2. H. Ludvigsen, M. Tossavainen \& M. Kaivola, Opt. Commun. 155, 180-186 (1998).

3. M. Puckett, D. Bose, K. Nelson, \& D. J. Blumenthal, CLEO: Science and Innovations (2019), pp. SM4O-1.

4. R. O. Behunin et. al. Phys. Rev. A 98, 023832 (2018).

5. H. Lee et. al. Nat. Photon. 6, 369-373 (2012).

6. L. Wu et. al. Opt. Lett. 45, 5129-5131 (2020).

7. H. Wang, L. Wu, Z. Yuan \& K. J. Vahala, arXiv:2010.09248 (2020).

8. J. Li, M.-G. Suh, \& K. J. Vahala, Optica 4, 346-348 (2017). 